Suzanne Guillemette Université de Sherbrooke

France Morin

Commission scolaire de la Pointe-de-l'île

Louise Simon

Université de Sherbrooke

\section{Une démarche de questionnement pour une gestion différenciée de l'activité éducative}

An inquiry approach for differentiated management of educational activities

\section{ésumé}

L'article propose une démarche de questionnement guidant une gestion différenciée de l'activité éducative chez des directions d'établissement. Cette démarche émerge d'une recherche-action réalisée auprès de deux cohortes de directeurs ou directrices de l'ordre d'enseignement primaire et secondaire dans deux commissions scolaires distinctes du Québec, sur une période de 15 mois. Les balises qui guident l'ajustement de pratique menant vers une gestion différenciée de l'activité éducative sont d'abord présentées pour ensuite rendre explicite la démarche de questionnement.

Mots-clés

Activité éducative, démarche de questionnement, différenciation, direction d'établissement, gestion différenciée, réussite

éducative

\section{Abstract}

This article proposes a questioning process contributing to differentiated leadership of the educational activities among school principals. This approach emerged from an action research conducted over a period of 15 months, with two groups of principals from primary and secondary levels within two school boards in Québec. The landmarks guiding the differentiated leadership of the educational activities are first presented, followed by the questioning process itself.

\section{Keywords}

Educational activity, questioning process, differentiation, school principal, differentiated leadership, academic success

\section{Introduction}

«À l'heure de transformations profondes et rapides des systèmes scolaires, la figure du directeur apparait comme l'une des références incontournables et sans doute déterminantes d'une adaptation de l'école à un monde nouveau »(Rich, 2010, p. 9). La complexité des responsabilités éducatives qui incombent à la direction d'établissement selon la Loi sur l'instruction publique (LIP) (Gouvernement du Québec, 2014) découle justement de cette réalité actuelle de l'éducation, ancrée dans un monde en constante transformation qui réclame un système éducatif plus inclusif où prévaut la culture de l'instant (Conseil supérieur de l'éducation, 2010; OCDE, 2010; Pont, Nusche et Moorman, 2008; Rich, 2010; Robin, Delory-Momberger et Boutinet, 2006). Cet état de fait commande à la direction de prendre un temps d'arrêt, d'effectuer une prise de recul, pour mieux se situer et ajuster ses pratiques de gestion de manière différenciée. L'article propose une démarche de questionnement qui favorise cette prise de recul au sein de l'équipe-école dans le but de répondre aux particularités du milieu. Il sera d'abord question du contexte de mouvance dans lequel œuvre la direction et de ce besoin de prise de recul, puis nous définirons la gestion différenciée de l'activité éducative. C'est par l'analyse de treize projets d'optimisation des activités éducatives ou pédagogiques qui ont été mis en œuvre dans le cadre d'une recherche-action avec des directeurs et directrices des ordres d'enseignement primaire et secondaire qu'il devient possible de dégager une démarche de questionnement et d'en discuter. 


\section{Responsabilités et complexité de la tâche de la direction d'établissement}

La direction d'établissement a la responsabilité de diriger l'élaboration d'un projet éducatif, d'un plan de réussite et d'une convention de gestion (Gouvernement du Québec, 2014) selon une perspective éducative. Cette responsabilité l'incite à réfléchir quant aux décisions et aux retombées plausibles dans le milieu (Langlois, 2002; Sergiovanni, 2007), dont la réussite éducative des élèves. Au Québec, cette réussite se décline selon trois axes : l'instruction, la socialisation et la qualification. Linstruction vise le développement du plein potentiel intellectuel, la socialisation met en valeur l'apprentissage du vivre ensemble dans une société sensible à la diversité culturelle, alors que la qualification vise l'insertion et l'intégration de tout citoyen à sa collectivité de manière active et autonome selon ses aspirations et son plein potentiel (Ministère de l'Éducation, du Loisir et du Sport [MELS], 2004).

Alors que la pratique de la direction d'établissement s'observe par les actions mises de l'avant selon des intentions (Zapata, 2004), sa responsabilité éducative vise dès lors les activités en réponse à cet objectif lié à la réussite éducative. Brassard et al. (2004) définissent justement l'expression gestion de l'activité éducative par " les activités d'aide à l'apprentissage des élèves et auxquelles s'intéresse d'une manière ou d'une autre le directeur dans l'exercice de sa fonction » (p. 480). Bien que la direction soit convaincue du bienfondé du regard éducatif dans les pratiques de gestion de l'activité éducative, elle évoque souvent la difficulté à prendre des moments de recul pour mieux évaluer les situations, compte tenu des tâches multiples et variées qui s'imposent à un rythme plutôt trépidant (Rich, 2010). Force est de constater que la direction gère trop souvent de manière fragmentée (Brassard et al., 2004; MELS, 2006) entre les tâches éducatives et administratives. Endrizzi et Thibert (2012) réaffirment quant à eux la pertinence d'une dynamique systémique afin de passer d'une posture d'école efficace à celle d'une école en amélioration constante qui «se préoccupe d'améliorer la performance de l'élève en se concentrant sur le processus d'enseignement-apprentissage» (p. 6). Dans une telle perspective intégratrice, les pratiques administratives et éducatives sont appelées à se jumeler (MELS, 2008). La gestion de l'activité éducative correspond alors aux actions que pose la direction dans une dynamique d'amélioration continue. Elle devient "l'aune à laquelle le processus administratif est revu et repensé » (Dupuis, 2004, p. 153). C'est pourquoi, la gestion de l'activité éducative se définit par :

Toute action qu'une direction d'établissement pose dans une visée intégratrice et systémique ayant une intention éducative au regard du projet d'établissement. [La gestion de l'activité éducative] s'observe par les actions que pose la direction [...] pour soutenir, guider et mobiliser l'équipe-école afin d'assurer, de stimuler ou d'enrichir les activités qui conduisent à la réussite de tous les élèves au sein de l'établissement selon les trois axes de la mission, à savoir : instruire, socialiser et qualifier. (Guillemette, 2014, p. 34)

La responsabilité éducative qui incombe à la direction s'appuie donc sur la façon de gérer de manière systémique l'ensemble des activités de l'établissement en réponse aux valeurs éducatives de la mission scolaire. Cette gestion exige de poser un regard macro-systémique (école, commission scolaire, communauté) et micro-systémique (classe, groupe, niveau, cycle ainsi que les interrelations entre ceux-ci). La gestion des activités éducatives de la direction, fondée sur une perspective éducative et systémique, occupe donc une place riche de sens et d'orientations pour assurer la réussite. Plusieurs recherches dont deux méta-analyses viennent justement affirmer la relation directe ou indirecte (Gurr, Drysdale et Mulford, 2006; Hallinger, 
2005; Leithwood, Day, Sammons, Harris et Hopkins, 2006; Marzano, Waters et McNulty, 2005) des pratiques de gestion de la direction sur la réussite des élèves. Ces recherches relèvent notamment toute l'importance pour une direction d'assurer un leadership solide et partagé, de mettre en place une culture axée sur l'enseignement-apprentissage, d'établir des attentes élevées, d'utiliser des données de rendement pour faire les suivis ainsi que d'instituer des méthodes pédagogiques adaptées à la réalité du milieu. Dans un tel contexte, la direction doit s'assurer de répondre aux particularités du milieu (Sergiovanni, 2007) afin de concrétiser la réussite de tout un chacun dans une perspective différenciée. Duke (2010) utilise l'expression « differenciating leadership » pour rendre explicite la nécessité pour la direction de différencier ses pratiques de gestion selon quatre profils distincts d'établissement avec un style de leadership à privilégier pour chaque profil. Guillemette (2014) présente plutôt huit balises, cinq faisant appel au processus de gestion et trois aux conditions de réalisation. Ces balises permettent à la direction de prendre un temps d'arrêt pour se situer par rapport aux particularités du milieu et ainsi mieux ajuster ses pratiques de gestion dans une perspective de différenciation.

Considérant la difficulté pour les directions à prendre un moment d'arrêt et à vivre une prise de recul, considérant que chaque établissement d'enseignement présente un profil d'hétérogénéité et des enjeux propres à son environnement, il parait sensé de remettre en question la façon dont la direction pose un regard analytique avec les membres de son équipe-école sur le contexte spécifique de son milieu dans le but d'ajuster ses pratiques de gestion de l'activité éducative. L'analyse de treize projets d'optimisation des activités éducatives ou pédagogiques menée dans le cadre d'une recherche-action permet de répondre à la question.

\section{Un cadre de référence}

Pour élucider cette question, il y a d'abord lieu de définir la notion de prise de recul menant vers un ajustement de pratique et ensuite la notion de gestion différenciée de l'activité éducative.

\section{Une prise de recul vers un ajustement de pratique}

La prise de recul s'observe par la capacité à prendre un temps d'arrêt pour mieux observer et analyser une situation et ainsi ajuster sa pratique. Selon Masciotra et Medzo (2009), toute situation se distingue d'un évènement par la façon dont ce dernier est appréhendé, perçu ou compris par un individu. Ainsi, la manière d'agir ou d'ajuster sa pratique s'inscrit dans un ensemble d'actes sociaux observables et repérables dans le but de produire un effet (Zapata, 2004). Dans une telle perspective, chaque pratique devient singulière, événementielle et contextuelle. L'ajustement de pratique exige alors de sortir d'une pensée linéaire, de poser un regard sur son agir pour adapter ou transformer les filtres personnels qui guident les intentions d'action (Guillemette et Simon, 2014). Par conséquent, l'ajustement de pratique repose sur la capacité d'identifier ses intentions d'action par rapport à une situation afin de mobiliser les ressources internes (les savoirs, les savoir-faire et les savoir-être) ainsi que les ressources externes (les individus et les outils) en réponse à une situation donnée, un évènement (Masciotra et Medzo, 2009). 


\section{La gestion différenciée de l'activité éducative}

À l'instar de Perrenoud (2012), le concept de différenciation renvoie « au refus de l'indifférence aux différences » (p. 37). Il propose pour y répondre de s'appuyer sur une analyse fine des causes de l'échec. Loin d'une approche individualisée, la différenciation évoque une philosophie d'intervention qui reconnait les différences et les potentialités de chacun comme richesse et source d'apprentissage au sein d'un collectif (Perrenoud, 1997; Przesmycki, 2004). Selon Przesmycki (2004) et Zakhartchouk (2001), la différenciation devient une avenue afin de répondre aux enjeux politique, sociologique et neuropsychologique. Sous l'angle de la gestion, la différenciation exige de la part de la direction de poser un regard plus large, c'est-à-dire sur le système-école et les sous-systèmes classe, cycle d'apprentissage, intercycle ou niveau. Pour améliorer la réussite, la direction cherche donc à comprendre les particularités du milieu, à dresser le portrait des élèves. La direction, avec l'équipe-école, identifie l'écart entre la situation actuelle, la réussite des élèves et la situation désirée, la hausse des taux de réussite. Pour identifier cet écart, la direction utilise cinq balises en lien avec le processus de gestion : les enjeux, les dispositifs de la différenciation, les étapes d'une démarche de projet, l'hétérogénéité et les domaines de gestion (Guillemette, 2014). Elle procède à une analyse fine du milieu, en cernant les enjeux politique (le droit à la réussite pour tous), sociologique (les caractéristiques socioculturelles et économiques du milieu) et neuropsychologique (les styles ou les particularités d'apprentissage des élèves). Des dispositifs (Hume, 2009; Przesmycki, 2004) associés à la différenciation sous l'angle de la gestion se définissent par le contenu (les objectifs visés au projet d'établissement), la structure (le type d'organisation scolaire nécessaire en fonction des encadrements légaux), les processus (la capacité de réflexivité des membres de l'équipe-école par le biais de la mobilisation des expertises de tous) et enfin le produit (le taux de réussite des élèves et le niveau de développement professionnel du personnel).

La direction mise aussi sur l'hétérogénéité du milieu, c'est-à-dire la complémentarité et la richesse des intervenants qui y ouvrent, et ce, pour mieux atteindre la visée éducative. Il s'agit alors d'identifier les compétences ainsi que les expertises des enseignants, des membres des services éducatifs complémentaires (orthopédagogue, psychologue, psychoéducateur, etc.) ainsi que des techniciens en éducation spécialisée. Dans son processus d'analyse, il devient tout aussi important de tenir compte du curriculum, du Programme de formation de l'école québécoise (PFEQ) et des encadrements légaux, dont la Loi sur l'instruction publique (LIP) et le régime pédagogique.

Sous l'angle de la gestion, les dispositifs de la différenciation viennent donc soutenir l'analyse du milieu, première étape de toute démarche de gestion de projet (Boutinet, 2005). Une telle analyse aide à mieux planifier, actualiser et réguler la démarche du projet doptimisation des activités éducatives et pédagogiques de létablissement. Conséquemment, cette démarche exige de poser un regard sur la façon de gérer l'école à partir des quatre domaines de gestion : les services éducatifs, l'environnement éducatif, les ressources humaines ainsi que la dimension administrative, c'est-à-dire les ressources matérielles et financières (MELS, 2008). Enfin, trois conditions de réalisation deviennent des balises essentielles à la mise en place d'une telle démarche à savoir se donner du temps, modéliser les stratégies d'écoute et de questionnement au sein de l'équipe-école et assurer le développement professionnel de tout le personnel (Guillemette, 2014). L'analyse de treize projets d'optimisation d'activités éducatives ou pédagogiques mis en œuvre dans le cadre d'une démarche de recherche-action permet de documenter comment une direction peut vivre cette prise de recul, et ce, à partir des huit balises énumérées qui guident une gestion différenciée de l'activité éducative. 


\section{Une méthodologie de recherche-action}

La démarche de recherche-action se qualifie par la relation étroite entre la pratique et la théorie. Elle favorise la recherche de sens par le développement de nouveaux savoirs (Reason et Bradbury, 2006). Elle privilégie une dynamique participative où le chercheur est appelé à se rendre sur le terrain pour réfléchir avec les praticiens par rapport à un objet de recherche (Guillemette et Savoie-Zajc, 2013; Savoie-Zajc, 2001). Dans le cas présent, l'opérationnalisation de la recherche s'est réalisée en filigrane à la mise en œuvre d'un modèle d'accompagnement collectif, échelonnée sur une période de 15 mois (octobre 2009 à janvier 2011) auprès de deux cohortes, chacune composée de neuf directions d'établissement des ordres d'enseignement primaire et secondaire. Chaque cohorte a été rencontrée 9 jours aux 5 à 6 semaines. Considérant les doubles fonctions, accompagnement et recherche, il y a lieu de distinguer d'abord l'opérationnalisation de la démarche d'accompagnement et ensuite l'opérationnalisation de la démarche de collecte et d'analyse des données.

\section{L'opérationnalisation de la démarche d'accompagnement}

Le modèle d'accompagnement collectif se distingue par la façon dont chaque direction conduit un projet d'optimisation des services éducatifs ou pédagogiques en réponse aux particularités de son milieu, tout en partageant son expérience avec les autres membres de sa cohorte (Guillemette, 2014). Chaque projet est présenté par une direction dans un but de le faire évoluer à partir d'une démarche d'écoute, de questionnement et de rétroaction. Ainsi, à tour de rôle, chaque direction devient tantôt la personne accompagnée et tantôt une personne accompagnatrice parmi le groupe. Divisées en trois moments, les rencontres prévoient d'abord un moment d'accueil qui sert à maintenir un lien de confiance et d'authenticité. Les dimensions théoriques évoquées à la rencontre précédente ainsi que les éléments coconstruits que l'on retrouve au compte rendu sont aussi validés. Vient ensuite la phase de réalisation qui se déploie selon quatre axes pour mieux analyser un projet : 1) remettre en question ou observer par rapport à une situation ou un agir; 2) analyser ou réfléchir à partir d'un questionnement pour mieux clarifier l'évènement et ainsi se situer; 3) échanger ou planifier pour identifier et choisir des pistes de solution et ainsi établir un plan d'action et 4) expérimenter et agir à partir du plan d'action établi. Cette dynamique interactive favorise la prise de recul dans le but d'ajuster les pratiques de gestion. La phase d'intégration et d'introspection permet enfin d'instaurer une dimension réflexive à la fois collective et individuelle. De manière individuelle, chaque direction est invitée à inscrire sur une fiche les éléments qu'elle retient, ceux qu'elle souhaite approfondir ainsi que ceux qu'elle peut ajuster dans sa pratique. De manière collective, chaque direction est invitée à s'exprimer sur ce qu'elle retient ou ce qui émerge à la suite des échanges et relativement à l'objet de la recherche.

\section{L'opérationnalisation de la démarche de collecte et d'analyse des données}

Le choix de l'échantillonnage porte sur des caractéristiques différentes et complémentaires, dont les caractéristiques territoriales : le type de commissions scolaires et le profil des participants qui forment les cohortes $\mathrm{CA}$ et $\mathrm{CB}$. Le choix du territoire est nuancé par sa situation géographique (CA : milieu urbain; $\mathrm{CB}:$ milieu rural), sa superficie (CA : +/- $700 \mathrm{~km}^{2} / \mathrm{CB}:+/-15000 \mathrm{~km}^{2}$ ) et son indice de milieu 
socioéconomique (IMSE : CA : 2 et CB : 9). L'échantillon des participants est constitué de manière aléatoire en fonction de l'intérêt des directions de l'ordre d'enseignement primaire ou secondaire à participer à la démarche d'accompagnement et de recherche. Chaque cohorte est formée de neuf directions où plus de $60 \%$ sont des femmes. Au sein de la cohorte $\mathrm{A}, 60 \%$ des participants ont de 5 à 10 ans d'expérience, $20 \%$ ont moins de 5 ans d'expérience et $20 \%$ en ont plus de 10 ans. Au sein de la cohorte B, $40 \%$ ont de 5 à 10 ans d'expérience, $40 \%$ ont plus de 10 ans d'expérience et seulement $20 \%$ ont moins de 5 ans d'expérience. Au total, 18 directions ont participé à la recherche.

Considérant la double posture formation et recherche, des critères de rigueur (Savoie-Zajc, 2001) assurent l'objectivation de la démarche alors que des invariants ou des spécificités s'en dégagent (Le Boterf, 2004). La cohérence systémique exige que le processus soit clairement explicité. La fiabilité et l'appropriation des données rendent explicite la façon de corroborer les données. Le respect des valeurs et des principes démocratiques exigent de tenir compte des propos des participants à la recherche. Enfin, la faisabilité de la recherche clarifie la façon de mener à terme la démarche de recherche. Le choix de l'échantillonnage ainsi que la triangulation des outils de collecte de données assurent la fiabilité et la cohérence systémique des données, c'est-à-dire la façon de corroborer les premiers invariants qui émergent entre trois outils de collecte de données tout en rendant explicite le contexte. Pour la présente étude, la transcription des échanges (plus de 250 heures) quant aux axes du modèle d'accompagnement par rapport à l'évolution des projets d'optimisation est un premier outil de collecte de données. Selon les critères du respect des valeurs et des principes de démocratie, chaque projet transcrit est lu et validé par son auteur, la direction concernée. Toujours dans une dynamique de triangulation, chaque projet est ensuite croisé aux deux autres outils de collecte de données complémentaires : les comptes rendus validés et les fiches d'introspection. Pour une même cohorte, l'évolution de chaque projet est analysée et croisée à l'évolution des autres projets. Des premiers invariants ou spécificités émergent alors de cette démarche d'analyse. Par une stratégie du regard croisé, ces invariants sont croisés et comparés aux invariants qui émergent de l'analyse de l'évolution des projets de la deuxième cohorte (CB). Cette stratégie assure l'objectivation des données qui émergent de la démarche d'analyse et permet d'identifier des invariants communs. Enfin, deux outils complémentaires sont privilégiés pour assurer l'objectivation des résultats de la recherche : un groupe de discussion à mi-parcours et des entrevues semi-dirigées au terme de la recherche. Un canevas d'entrevue de groupe ainsi qu'un canevas d'entrevue semi-dirigée permettent de corroborer les dires des participants par rapport à l'évolution de leur projet. Au total, treize projets ont été transcrits, croisés aux autres outils et analysés pour permettre de cerner la façon dont les directions se questionnent avec les membres de l'équipe-école à partir des balises de la différenciation et de l'ajustement de leurs pratiques de gestion. Afin de faire des liens avec les critères de fiabilité et de faisabilité, un journal de bord est utilisé par la personne accompagnatrice et chercheuse. Elle y note les éléments de convergence ou d'incohérence qui peuvent surgir quant à lévolution des projets par rapport aux éléments qui apparaissent par le biais des autres outils de collecte de données. L'analyse de l'évolution de chaque projet et de leur contenu utilise la démarche de thématisation continue (Paillé et Mucchielli, 2008) qui commande à titre de premières balises les enjeux, les dispositifs de la différenciation et les domaines de gestion, et ce, en lien avec les quatre temps d'une démarche de projet (Boutinet, 2005). S'y greffe l'identification des intentions d'action : 1) analyser : se questionner pour comprendre la réalité du milieu et clarifier les ambigüités; 2) planifier : prioriser et organiser les actions à mettre de l'avant, en explorant de nouvelles perspectives, 
en identifiant la façon de réduire l'écart entre la situation actuelle et la situation désirée et en identifiant les priorités à mettre de l'avant; 3) actualiser : agir en fonction des décisions prises; 4) réguler : observer ou évaluer, à l'aide d'outils de veille, les retombées réelles des pratiques de gestion sur la diminution de l'écart entre la situation de départ et celle atteinte.

\section{Une démarche de questionnement pour une gestion différenciée}

L'analyse des projets d'optimisation, croisés aux fiches d'intégration ainsi qu'aux comptes rendus, permet d'identifier toute la place que prennent les enjeux (sociologique, politique ou neuropsychologique) ainsi que les dispositifs de la différenciation (contenu, processus, structure et produit). En complément, l'analyse relative aux intentions d'action des directions, croisées aux étapes de toute démarche de projet (Boutinet, 2005), permet de dégager une démarche de questionnement susceptible de faire cheminer les équipes-écoles. Par exemple, au stade de l'analyse de la situation, une direction questionne la place qu'occupe l'enjeu neuropsychologique : les profils ou les spécificités d'apprentissage des élèves au sein de l'établissement à volet alternatif (projet de CA07), une deuxième direction questionne plutôt la dimension sociologique (projet de CB12) pour mieux répondre aux spécificités de son école qui se situe en milieu éloigné et défavorisé (IMSE de 9) alors qu'une troisième direction, cette fois en milieu urbain, s'interroge sur la façon d'assurer l'interdisciplinarité entre le Programme de l'école internationale et le Programme de formation de l'école québécoise pour permettre la réussite de tous ses élèves, ce qui illustre des enjeux politiques (projet CA08). Quelques questions viennent illustrer le propos :

\section{Enjeu neuropsychologique :}

- Quel est le portrait des élèves?

- Les styles d'apprentissage;

- $\quad$ Les difficultés d'apprentissage;

- Les talents ou les forces des élèves.

\section{Enjeu sociologique}

- Comment qualifie-t-on l'indice de défavorisation du milieu?

- Quel est le portrait socioéconomique et socioculturel du milieu?

- Le niveau de scolarité de la mère;

- La proportion de parents actifs dans le milieu;

- La composition ethnique des élèves du milieu;

- La langue parlée à la maison.

\section{Enjeu politique}

- Quel est le portrait de la réussite éducative par rapport à l'axe « instruire »?

- Les taux de réussite en numératie ou en littératie;

- Au sein des autres domaines disciplinaires;

- Quel est le portrait de la situation par rapport à l'axe « socialiser »?

- Pour développer les habiletés sociales des élèves;

- Pour développer de saines habitudes de vie chez les élèves;

- En prévention ainsi qu'en traitement de la violence; 
- Quel est le portrait de la situation par rapport à l'axe « qualifier »?

- Le taux de diplomation;

- Le taux de décrochage;

- Le taux de persévérance;

- Le nombre d'élèves dans un parcours menant à un métier;

- Les activités éducatives qui permettent aux élèves de découvrir leurs intérêts, leurs forces ou leurs passions.

Lors des échanges entre les directions, une mise en garde vient mettre en évidence l'importance de garder un certain équilibre entre les trois enjeux afin de ne pas sombrer dans une seule façon de faire. Par exemple, à trop vouloir répondre exclusivement aux particularités de chaque élève, les directions prennent conscience que les enseignants sortent de la dynamique de différenciation pour travailler dans une perspective d'individualisation. Aux dires d'une direction, "la limite de la différenciation [exige de] respecter l'équilibre entre le socio, le neuro et le politique, accepter qu'il y ait des limites» alors que pour une autre direction "à vouloir se centrer exclusivement sur le portrait spécifique de chaque élève, l'enjeu neuropsychologique, il y a un danger de passer à côté des enjeux sociologiques ou politiques rattachés à notre milieu».

$\mathrm{Au}$-delà du questionnement par rapport aux enjeux relatifs à chaque milieu ou à chaque projet, il appert que le questionnement itératif ayant comme repères les dispositifs de la différenciation - structure, contenu, processus et produit - vient soutenir la prise de recul quant à l'analyse, à la planification, à l'agir et à la régulation. Ainsi, dans un esprit de synthèse et à titre d'exemple, ces quelques questions mises de l'avant avec les membres de l'équipe-école soutiennent l'ajustement des pratiques de gestion.

1. Le contenu : objectifs visés au projet d'établissement.

- Sur quoi allons-nous collaborer?

- Quels sont nos intentions ou nos objectifs?

- En quoi répondent-ils aux besoins des élèves?

- $\quad$ Sur quelles pratiques éducatives ou pédagogiques voulons-nous réfléchir?

- Quelles sont les connaissances ou les compétences que nous possédons?

- Quelles sont celles à développer pour atteindre nos objectifs?

2. La structure : le type d'organisation scolaire nécessaire, en tenant compte des encadrements légaux.

- Comment voulons-nous nous organiser?

- Comment allons-nous constituer les équipes de travail afin que chacun puisse contribuer et apprendre?

- Comment peut-on adapter la grille horaire?

- Quels seront les moments de rencontres? Leur fréquence?

- Quel est notre échéancier?

- Quel est le rôle de chacun? 
3. Le processus : la capacité de réflexivité des membres de l'équipe-école selon la mobilisation des expertises de tous.

- Comment voulons-nous collaborer?

- Quelles sont les questions incontournables?

- Comment s'assurer de l'engagement cognitif et de la qualité de la dynamique interactive de notre groupe de travail?

- Quelles sont les pratiques pédagogiques à privilégier en classe?

- Comment peut-on mobiliser les expertises existantes dans notre milieu : enseignant, orthopédagogue, psychologue, etc. ?

- Quelles sont les stratégies cognitives et métacognitives à privilégier en classe?

- Quelles sont les pratiques éducatives à privilégier en classe et au sein de l'école?

4. Le produit : le taux de réussite des élèves, le niveau de développement professionnel du personnel, etc.

- Quels sont les résultats à obtenir?

- Quels sont les domaines disciplinaires visés?

- Quels sont les besoins de formation ou de développement professionnel du personnel pour répondre aux attentes?

- Quelle est la manière de monitorer l'ajustement des pratiques pédagogiques (classe) ou des pratiques éducatives (école et classe)?

Selon les directions associées à la recherche, ce questionnement favorise la réflexion en nommant les particularités du milieu ainsi que la mobilisation possible des expertises existantes au sein du personnel pour y répondre (Guillemette, 2014; Sergiovanni, 2007). Les questions permettent justement de poser un regard systémique (Endrizzi et Thibert, 2012), d'abord de manière macro, l'établissement d'enseignement et ensuite, de manière micro, par rapport aux sous-systèmes, le $2^{\mathrm{e}}$ cycle de l'établissement par exemple. À juste titre, une direction précise que "l'on gère à deux niveaux [...] l'équipe de profs et [...] les élèves sous la responsabilité des profs. " Cette démarche de questionnement soutient la manière d'ajuster les pratiques. McTighe et Wiggins (2013) qualifient ce type de questionnement de « higher order of thinking ", un niveau supérieur ou méta de réflexion. Aux dires des directions ayant participé à la recherche, il s'agit « de prendre une distance, [de] réfléchir et [de] se placer au-dessus du problème » ou à titre d'analogie, de « se placer au balcon ». C'est alors que la direction sort d'une pensée linéaire, adapte ou transforme ses pratiques de gestion, à savoir « il est important de les voir dans leur globalité [...] planifier et faire des boucles de régulation ". Considérant cette nouvelle compréhension de la réalité, la direction est davantage en mesure d'explorer de nouvelles façons de faire et de planifier, avec les membres de l'équipe, des objectifs réalistes quant à l'identification de pratiques éducatives ou pédagogiques adaptées au contexte de l'établissement. Une direction précise toute l'importance de « ramener les membres de l'équipe à se recentrer sur ce que nous attendons d'eux et sur ce qu'il est possible de faire ». Dès lors, la direction peut ajuster ses pratiques de gestion éducative de manière différenciée. 


\section{Conclusion}

Les résultats de la recherche démontrent la complexité de la tâche de toute direction qui se préoccupe de bien comprendre les particularités du milieu et envisage la mobilisation possible des expertises des membres du personnel qui y ouvrent afin d'améliorer la réussite éducative des élèves dans une perspective d'amélioration des pratiques (Endrizzi et Thibert, 2012). La démarche de questionnement qui émerge de la recherche guide la prise de recul et assure un leadership partagé selon une culture axée sur l'enseignement-apprentissage (Gurr et al., 2006; Hallinger, 2005; Leithwood et al., 2008; Marzano et al., 2005). Ce questionnement permet justement de recentrer les efforts de chacun sur des attentes élevées pour assurer la réussite éducative, raison d'être d'un établissement scolaire. La dimension différenciée des pratiques de gestion de toute activité éducative repose alors sur la capacité de la direction d'établissement à poser un regard stratégique sur l'hétérogénéité du milieu dans lequel elle œuvre, en tant que richesse (Perrenoud, 1997; Przesmycki, 2004) pour répondre au projet de l'établissement (Boutinet, 2005). Pour y parvenir, la démarche de questionnement devient un outil, voire une ressource indispensable pour guider l'amélioration des pratiques de gestion.

\section{Références}

Boutinet, J.-P. (2005). Anthropologie du projet. Paris : Presses universitaires de France.

Brassard, A., Cloutier, M., De Saedeleer, S., Corriveau, L., Fortin, R., Gélinas, A. et Savoie-Zajc, L. (2004). Rapport à l'activité éducative et identité professionnelle chez les directeurs d'établissement des ordres d'enseignement préscolaire et primaire. Revue des sciences de l'éducation, 30(3), 487-508. http://dx.doi.org/10.7202/012079ar

Conseil supérieur de l'éducation. (2010). Conjuguer équité et performance en éducation, un défi de société. Rapport sur l'état et les besoins de l'éducation 2008-2010. Repéré à https://www.cse.gouv.qc.ca/fichiers/documents/publications/CEBE/50-0192.pdf

Duke, D. L. (2010). Differentiating school leadership: Facing the challenges of practice. Thousand Oaks, CA : Corwin Press.

Dupuis, P. (2004). L'administration de l'éducation : quelles compétences? Éducation et francophonie, 32(2), 133-157. Repéré à http://www.acelf.ca/c/revue/pdf/Ladministrationdeleducation.pdf

Endrizzi, L. et Thibert, R. (2012). Quels leaderships pour la réussite de tous les élèves? Dossier de veille de l'Institut français de l'éducation, (73). Repéré à http://tinyurl.com/pzx2xjz

Gouvernement du Québec. (2014). Loi sur l'instruction publique. Repéré à http://tinyurl.com/5el4p8

Guillemette, S. (2014). Une gestion différenciée de l'activité éducative en milieu scolaire. Ajustement de pratique : modèle d'accompagnement collectif auprès de chefs ou de directions d'établissement scolaire. Berlin: Presses Académiques Francophones.

Guillemette, S. et Simon, L. (2014). Dispositifs d'un modèle d'accompagnement collectif qui guident la réflexivité chez des directions d'établissement en milieu scolaire. Revue de l'analyse de pratiques professionnelles, 3, 13-27. http://www.analysedepratique.org/?p=1223

Guillemette, S. et Savoie-Zajc, L. (2013). La recherche-action et ses rapports de coconstruction de savoirs et de formation dans une perspective de professionnalisation entre acteurs praticiens et chercheurs. Formation et profession: revue scientifique internationale en éducation. 20(3), 41-53. http://formation-profession.org/pages/article/20/3/7

Gurr, D., Drysdale, L. et Mulford, B. (2006). Models of successful principal leadership. School Leadership E̋ Management, 26(4), 371-395. http://dx.doi.org/10.1080/13632430600886921 
Hallinger, P. (2005). Instructional leadership and the school principal : A passing fancy that refuses to fade away. Leadership E' Policy in Schools, 4(3), 221-239. http://dx.doi.org/10.1080/15700760500244793

Hume, K. (2009). Comment pratiquer la pédagogie différenciée avec de jeunes adolescents? La réussite scolaire pour tous. Bruxelles : De Boeck.

Langlois, L. (2002). Le leadership en éducation : plusieurs regards, une même passion. Montréal, QC : Chenelière Éducation.

Le Boterf, G. (2004). Construire les compétences individuelles et collectives, la compétence n'est plus ce quelle était. Paris : Organisation.

Leithwood, K., Day, C., Sammons, P., Harris, A. et Hopkins, D. (2006). Seven strong claims about successful school leadership. Repéré à http://dera.ioe.ac.uk/6967/1/download\%3Fid\%3D17387\%26filename\%3Dseven-claims-about-successfulschool-leadership.pdf

Marzano, R. J., Waters, T. et McNulty, B. A. (2005). A school leadership that works, from research to results. Alexandria, VA : Association for Supervision and Curriculum Development.

Masciotra, D. et Medzo, F. (2009). Développer un agir compétent : vers un curriculum pour la vie. Bruxelles : De Boeck.

McTighe, J. et Wiggins, G. P. (2013). Essential questions : opening doors to student understanding. Alexandria, VA : Association for Supervision and Curriculum Development.

Ministère de l'Éducation, du Loisir et du Sport (MELS). (2004). Programme de formation de l'école québécoise, Enseignement secondaire, premier cycle (nº3-00690). Québec, QC : Gouvernement du Québec.

Ministère de l'Éducation, du Loisir et du Sport (MELS). (2006). Études pratiques de soutien et d'accompagnement des nouvelles directions d'établissement ( $\mathrm{n}^{\circ}$ 2006-06-00010). Repéré à

http://www.education.gouv.qc.ca/fileadmin/site web/documents/dpse/EtudePratiqueSoutienAccomp int f.pdf

Ministère de l'Éducation, du Loisir et du Sport (MELS). (2008). La formation à la gestion d'un établissement d'enseignement, les orientations et les compétences professionnelles. Repéré à http://www.education.gouv.qc.ca/fileadmin/site web/ documents/reseau/formation titularisation/07-00881.pdf

OCDE. (2010). Les clés de la réussite: Impact des connaissances et compétences à l'âge de 15 ans sur le parcours des jeunes Canadiens. Repéré à http://www.oecd.org/pisa/44576147.pdf

Paillé, P. et Mucchielli, A. (2008). L'analyse qualitative en sciences humaines et sociales. Paris : Armand Colin.

Perrenoud, P. (1997). Pédagogie différenciée : des intentions à l'action. Issy-les-Moulineaux : ESF.

Perrenoud, P. (2012). L’organisation du travail, clé de toute pédagogie différenciée. Issy-les-Moulineaux : ESF.

Pont, B., Nusche, D. et Moorman, H. (2008). Améliorer la direction des établissements scolaires (vol. 1 : Politiques et pratiques). Repéré à http://www.oecd.org/fr/edu/scolaire/44374906.pdf

Przesmycki, H. (2004). La pédagogie différenciée. Paris : Hachette Éducation.

Reason, P. et Bradbury, H. (dir.) (2006). The Sage handbook of action research : participative inquiry and practice. Thousand Oaks, CA : SAGE Publications.

Rich, J. (2010). Les nourveaux directeurs d'école, repenser l'encadrement des établissements scolaires. Bruxelles : De Boeck.

Robin, J.-Y., Delory-Momberger, C. et Boutinet, J.-P. (2006). Un tournant épistémologique : des récits de vie aux entretiens carriérologiques. Paris : L'Harmattan.

Savoie-Zajc, L. (2001). La recherche-action en éducation : ses cadres épistémologiques, sa pertinence, ses limites. Dans M. Anadon (dir.), Nouvelles dynamiques de recherche en éducation (p.15-49). Québec, QC : Presses de l'Université Laval.

Sergiovanni, T.J. (2007). Rethinking leadership : a collection of articles ( $2^{\mathrm{e}}$ éd.). Thousand Oaks, CA : Corwin Press. 
Zakhartchouk, J.-M. (2001). Au risque de la pédagogie différenciée. Paris : Institut national de recherche pédagogique.

Zapata, A. (2004). L'épistémologie des pratiques, pour l'unité du savoir. Paris : L'Harmattan.

\section{Pour citer cet article}

Guillemette, S., Morin, F. et Simon, L. (2015). Une démarche de questionnement pour une gestion différenciée de l'activité éducative. Formation et profession, 23(3), 45-56. http://dx.doi.org/10.18162/fp.2015.278 